\title{
On the flavour dependence of polarized sea in the nucleon *
}

\author{
Jan Bartelski \\ Institute of Theoretical Physics, Warsaw University, \\ Hoża 69, 00-681 Warsaw, Poland. \\ Stanisław Tatur \\ Nicolaus Copernicus Astronomical Center, \\ Polish Academy of Sciences, \\ Bartycka 18, 00-716 Warsaw, Poland.
}

preprint CAMK No. 328

\begin{abstract}
We performed a phenomenological fit in order to get quark parton polarized distributions in the nucleon. All data on inclusive and semiinclusive spin asymmetries measured on nucleon targets were used. We present the results for the flavour dependence of polarized sea inside a nucleon. An excellent agreement between inclusive and semi-inclusive data on polarized structure functions was found in our model.
\end{abstract}

*Work supported in part by the KBN-Grant PB 2-P03B-065-10. 
Recently the SMC collaboration has presented [1] the experimental results of semi-inclusive spin asymmetries for positively and negatively charged hadrons. A lot of earlier results for the polarized inclusive deep inelastic scattering on protons, neutrons $\left({ }^{3} \mathrm{He}\right)$ and deuterons [2, 3] are also available. There were also theoretical attempts to get the polarized parton distributions using these measured asymmetries [4]. The SMC group has tried to determine (using inclusive and semi-inclusive hadron asymmetries) spin distributions for valence (up and down) quarks and for non-strange sea quarks. In this paper we want to extend our previous determination of polarized parton distributions [5, 6] obtained from the inclusive spin asymmetries by taking into account new semi-inclusive data. With the new hadronic semi-inclusive asymmetries we will try to get more information about flavour structure of polarized sea. Our idea, as before, is to use the unpolarized parton distributions (see for example [7, 8]) and to split $q(x)\left(q(x)=q^{+}(x)+q^{-}(x)\right.$ ) in order to get the polarized ones, i.e. $q^{+}(x)$ and $q^{-}(x)$. With such a procedure one has simultaneous description of spin and spin averaged structure functions.

The latest version of the MRS (Martin, Roberts and Stirling [8]) fit to unpolarized parton distributions yields for the valence quarks (at $Q^{2}=4 \mathrm{GeV}^{2}$ ):

$$
\begin{aligned}
& u_{v}(x)=2.704 x^{-0.407}(1-x)^{3.96}(1-0.76 \sqrt{x}+4.20 x), \\
& d_{v}(x)=0.251 x^{-0.665}(1-x)^{4.41}(1+8.63 \sqrt{x}+0.32 x),
\end{aligned}
$$

whereas for the antiquarks from the sea one has:

$$
\begin{aligned}
& 2 \bar{u}(x)=0.392 M(x)-\delta(x), \\
& 2 \bar{d}(x)=0.392 M(x)+\delta(x), \\
& 2 \bar{s}(x)=0.196 M(x), \\
& 2 \bar{c}(x)=0.020 M(x) .
\end{aligned}
$$

The singlet contribution (which gives the total polarization of the nucleon sea) is:

$$
M(x)=1.74 x^{-1.067}(1-x)^{10.1}(1-3.45 \sqrt{x}+10.3 x) .
$$

For the isovector part of quark sea and for gluon distribution we have, respectively:

$$
\begin{gathered}
\delta(x)=0.043 x^{-0.7}(1-x)^{10.1}(1+64.9 x) \\
G(x)=1.51 x^{-1.301}(1-x)^{6.06}(1-4.14 \sqrt{x}+10.1 x) .
\end{gathered}
$$


We assume, in an analogy to the unpolarized case, that the polarized quark distributions are of the form: $x^{\alpha}(1-x)^{\beta} P_{2}(\sqrt{x})$, where $P_{2}(\sqrt{x})$ is a second order polynomial in $\sqrt{x}$ and the asymptotic behaviour for $x \rightarrow 0$ and $x \rightarrow 1$ (i.e. the values of $\alpha$ and $\beta$ ) are the same (except for $\Delta M$ ) as in unpolarized case. We split the numerical constants (coefficients of $P_{2}$ polynomial) in eqs. (1, 3 and 4$)$ in two parts in such a manner that we get positive defined distributions. Our expressions for $\Delta q(x)\left(\Delta q(x)=q^{+}(x)-\right.$ $\left.q^{-}(x)\right)$ for valence quarks are:

$$
\begin{aligned}
& \Delta u_{v}(x)=x^{-0.407}(1-x)^{3.96}\left(a_{1}+a_{2} \sqrt{x}+a_{3} x\right), \\
& \Delta d_{v}(x)=x^{-0.665}(1-x)^{4.41}\left(b_{1}+b_{2} \sqrt{x}+b_{3} x\right) .
\end{aligned}
$$

For a moment we will not take into account polarized gluons, i.e. we put $\Delta G=0$.

In our earlier fits we had assumed that the total sea polarization $(\Delta M)$ has no term behaving like $x^{-1.067}$ at small $x$ (we assumed that all distributions are integrable) and only one (as in unpolarized case) is determinig all sea contributions for different flavours: up, down, strange and charm. That assumption was used to reduce the number of unknown parameters but it was very restrictive. The strenght of strange quark sea polarization $\Delta s$ was practically determined by the value of $a_{8}$. With high value of $\Delta s$ (or equivalently $\Delta M) \Delta \bar{u}$ and $\Delta \bar{d}$ were also forced to be big (especially $\Delta \bar{d}$ ) because $\Delta \delta$ (see eq.(2)) was negative. That was rather unnatural. With the additional new data on semi-inclusive hadron asymmetries we can relax this assumption and see what are the polarized sea contributions for different flavours. Now we propose that for different flavours polarized sea distributions are different (except for the charm flavour but this is completely unimportant numerically), namely:

$$
\Delta M_{i}(x)=x^{-0.567}(1-x)^{10.1}\left(c+c_{i} \sqrt{x}\right) .
$$

In particular we have for sea contribution of different flavours:

$$
\begin{aligned}
\Delta u_{M}=\Delta \bar{u}(x) & =0.196 \Delta M_{u}(x)-0.5 \Delta \delta(x) \\
\Delta d_{M}=\Delta \bar{d}(x) & =0.196 \Delta M_{d}(x)+0.5 \Delta \delta(x), \\
\Delta s_{M}=\Delta \bar{s}(x) & =0.098 \Delta M_{s}(x) \\
\Delta c_{M}=\Delta \bar{c}(x) & =0.010 \Delta M_{s}(x)
\end{aligned}
$$


where $\Delta q_{M}(x)=\Delta q(x)-\Delta q_{v}(x)-\Delta \bar{q}(x)$ and:

$$
\Delta \delta(x)=x^{-0.7}(1-x)^{10.1} d(1+64.9 x) .
$$

We assume that the sea contributions for quarks and antiquarks are equal (this assumption will be reconsidered later). In order to get the unknown parameters in the expressions for polarized quark distributions at $Q^{2}=4 \mathrm{GeV}^{2}$ (see eqs.(6-9)) we make a fit to the experimental data on inclusive spin asymmetries for proton, neutron and deuteron targets and semi-inclusive hadron asymmetries on proton and deuteron. The theoretical expressions for inclusive spin asymmetries are given by:

$$
A_{1}\left(x, Q^{2}\right)=\frac{\sum_{q} e_{q}^{2} \Delta q\left(x, Q^{2}\right)}{\sum_{q} e_{q}^{2} q\left(x, Q^{2}\right)}(1+R)
$$

where $e_{q}$ is the charge of the q-flavoured quark. The ratio $R=\sigma_{L} / \sigma_{T}$ (which vanishes in the Bjorken limit) is taken from [9]. The expression for deuteron asymmetry is considered to be the sum of the corresponding expressions for proton and neutron corrected for the D-state portion in the deuteron $\left(p_{D}\right)$, equal to $(5 \pm 1) \%$ [2]. The expressions for semi-inclusive spin asymmetries for the production of positive and negative hadrons are given by:

$$
A_{1}^{+(-)}\left(x, Q^{2}\right)=\frac{\sum_{q, h} e_{q}^{2} \Delta q\left(x, Q^{2}\right) D_{q}^{h}\left(Q^{2}\right)}{\sum_{q, h} e_{q}^{2} q\left(x, Q^{2}\right) D_{q}^{h}\left(Q^{2}\right)}(1+R)
$$

where $D_{q}^{h}\left(Q^{2}\right)=\int_{0.2}^{1} d z D_{q}^{h}\left(z, Q^{2}\right)$. Following the usual convention all quark distributions refer to the proton. To reduce the number of independent fragmentation functions we use the same relations as in ref. [1] and 10. From eq.(11) one can see that the presence of different weights $\left(D_{q}^{h^{ \pm}}\right)$enables to extract different combinations of $\Delta q$ than in the inclusive spin asymmetries. First of all we investigate what happens when we add measured semi-inclusive hadron asymmetries to the sample of inclusive data on spin structure of the nucleon. Taking the parameters of the fit from our previous paper [6] we can calculate that the contribution of semi-inclusive data from SMC group gives $\chi_{S M C}^{2}=62.5$ (for 48 experimental points) and from EMC [3] $\chi_{E M C}^{2}=20.2$ (for 10 points) and together with $\chi_{\text {incl }}^{2}=96.5$ we get $\chi_{\text {total }}^{2} \cong 179$. We can also compare our predictions for $A_{1 p}^{+(-)}$and $A_{1 d}^{+(-)}$with the measured SMC and EMC results. When we make fit to all the data (inclusive and semiinclusive) the total value is $\chi_{\text {all }}^{2}=178.5$ so we do not gain much in $\chi^{2}$. The 
fitted parameters are very close to the parameters obtained with inclusive data only (the new calculated values for $A_{1 p}^{+(-)}$and $A_{1 d}^{+(-)}$also change very little.) That means that the new semi-inclusive data are consistent with inclusive data. We assume that the spin asymmetries do not depend on $Q^{2}$ (it is only our first order approximation) what is suggested by the experimental data [2]. We hope that numerically our results at $Q^{2}=4 \mathrm{GeV}^{2}$ will not change much if the evolution of $F_{1}$ and $g_{1}$ functions will be taken into account. The semi-inclusive hadron asymmetries have relatively big errors (there are also uncertainties connected with the quark fragmentation functions) and also it is not clear how reliable is the fit to $\mathrm{R}$ function for small $x$. Taking all that into account we will take crude approximation neglecting the $Q^{2}$ dependence of the spin asymmetries.

The obtained quark distributions $\Delta u_{v}(x), \Delta d_{v}(x), \Delta M_{u}(x), \Delta M_{d}(x)$, $\Delta M_{s}(x)$ and $\Delta \delta(x)$ can be used to calculate first moments. For a given $Q^{2}$ we can write the relations:

$$
\begin{aligned}
& \Gamma_{1}^{p}=\frac{4}{18} \Delta u+\frac{1}{18} \Delta d+\frac{1}{18} \Delta s+\frac{4}{18} \Delta c, \\
& \Gamma_{1}^{n}=\frac{1}{18} \Delta u+\frac{4}{18} \Delta d+\frac{1}{18} \Delta s+\frac{4}{18} \Delta c,
\end{aligned}
$$

where $\Delta q=\int_{0}^{1} \Delta q(x) d x$ and $\Gamma_{1}=\int_{0}^{1} g_{1}(x) d x$.

We define other combinations of integrated quark polarizations:

$$
\begin{aligned}
a_{3} & =\Delta u-\Delta d, \\
a_{8} & =\Delta u+\Delta d-2 \Delta s, \\
\Delta \Sigma & =\Delta u+\Delta d+\Delta s,
\end{aligned}
$$

Such results for the integrated quantities (calculated at $4 \mathrm{GeV}^{2}$ ) after taking into account known QCD corrections (see e.g. Ref.[11]) could be compared with axial-vector coupling constants $g_{A}$ and $g_{8}$ known from neutron $\beta$-decay and hyperon $\beta$-decays (in the last case one needs $S U(3)$ symmetry). In our paper $Q^{2}$ is constant and takes the value $4 \mathrm{GeV}^{2}$. Using experimental numbers [12] we expect that $a_{3}\left(4 \mathrm{GeV}^{2}\right)=1.11$ and $a_{8}\left(4 \mathrm{GeV}^{2}\right)=0.51 \pm 0.03$. As we have made in the previous fits in order to stabilize the determination of parameters we assume in addition that $a_{8}=0.51$ (with 0.1 as fictive theoretical error). 
We get the following values of our parameters (describing the polarized quark distributions in eqs.(6-9)) from the fit to all existing data for spin asymmetries inclusive and semi-inclusive:

$$
\begin{aligned}
& a_{1}=1.07, \quad a_{2}=-4.15, \quad a_{3}=11.9 \\
& b_{1}=-0.25, \quad b_{2}=1.02, \quad b_{3}=3.18 \\
& c_{u}=-0.90, \quad c_{d}=3.44, \quad c_{s}=-3.90 \\
& c=-0.37, \quad d=-0.04
\end{aligned}
$$

For this fit we get $\chi^{2} / N_{D F}=1.12$ We will not present the comparison of our fit with the experimental inclusive asymmetries for proton, neutron and deuteron targets because they are not very much different from the previous figures. The comparison of the semi-inclusive spin asymmetries for positive and negative hadrons on proton and deuteron targets with experimental points obtained by SMC [1] and EMC [3] groups in CERN are given in figures $1 \mathrm{a}, \mathrm{b}$ and $2 \mathrm{a}, \mathrm{b}$. Polarized quark distributions for up and down valence quarks and up, down and strange sea quarks are presented in figures 3a-3f. The obtained quark distributions lead to the following integrated quantities: $\Delta u=0.74\left(\Delta u_{v}=0.71\right), \Delta d=-0.52\left(\Delta d_{v}=-0.39\right)$ and $\Delta s=-0.14$. These numbers lead to the following predictions: $\Gamma_{1}^{p}=0.125, \Gamma_{1}^{n}=-0.084$, $a_{3}=1.25, \Delta \Sigma=0.08, \Delta M=-0.24$ The total sea contribution was strongly reduced in comparison with the model considered before. We have small positively polarized sea for up quark and stronger negatively polarized sea for down and strange quarks. As was already stressed in [5, 6] the behaviour at small $x$ of our polarized quark distributions is determined by the unpolarized ones and these do not have the expected theoretically Regge type behaviour (which is also used by experimental groups to extrapolate results to small values of $x$ ). Unfortunately, some of the quantities change rapidly for $x<0.003$ (e.g. down quark distribution). We present quantities integrated over the region from $x=0.003$ to $x=1$ (it is practically integration over the region which is covered by the experimental data, except of noncontroversial extrapolation for highest $x$ ). The corresponding quantities are: $\Delta u_{v}=0.66$, $2 \Delta \bar{u}=0.03,(\Delta u=0.69), \Delta d_{v}=-0.29,2 \Delta \bar{d}=-0.08,(\Delta d=-0.37)$, $\Delta s=-0.12, \Delta M=-0.17$ and $\Gamma_{1}^{p}=0.123, \Gamma_{1}^{n}=-0.054, a_{3}=1.06$, $\Delta \Sigma=0.20$.

The above given values extrapolated to $x=0$ using Regge type behaviour $x^{-0.25}$ for small $x$ give: $\Delta u_{v}=0.70,2 \Delta \bar{u}=0.02,(\Delta u=0.72), \Delta d_{v}=-0.33$, 
$2 \Delta \bar{d}=-0.10,(\Delta d=-0.43), \Delta s=-0.13, \Delta M=-0.21$ and $\Gamma_{1}^{p}=0.126$, $\Gamma_{1}^{n}=-0.066, a_{3}=1.07, \Delta \Sigma=0.16$,

Our results can be compared with that for the non strange sea polarization $\Delta \bar{q}=-0.02$ obtained by SMC group [1] (under the assumption $\Delta \bar{u}=\Delta \bar{d}$ and assumed value for $\Delta s=-0.12$ ). Also our valence up and down quark contributions are different from those presented in [1].

When we use this model (with flavour dependent polarization of the sea contributions) to make a fit to the subset of the data on inclusive spin asymmetries we get the fitted parameters and results very close to the considered model with $\chi^{2} / N_{D F}=1.01$. For example we get $2 \Delta \bar{u}=0.05,2 \Delta \bar{d}=-0.09$ and $2 \Delta \bar{s}=-0.12$ in $0.003 \leq x \leq 1$ region. So it was possible to obtain the information about flavour dependence of polarized sea without taking into account data on semi-inclusive hadron spin asymmetries only. That shows a consistency of both sets of data (inclusive and semi-inclusive) with our assumption of flavour dependence of polarized sea. When we use instead of the MRS fit used in this paper another version called A' [8] the similar results are obtained. The results practically do not depend on the precise form of the MRS fit for unpolarized parton distributions used as a starting point for polarized parton distributions. As in the previous papers [5, 6] we have also tried to include polarized gluons along the line of [13] assuming for the gluon distribution:

$$
\Delta G(x)=x^{-0.801}(1-x)^{6.06}\left(d_{1}+d_{2} \sqrt{x}\right),
$$

with a new $d_{1}$ and $d_{2}$ constants which have to be fitted. The appearance of non-zero gluonic distribution affects our formulas only through the substitution: $\Delta q \Rightarrow \Delta q-\frac{\alpha_{s}}{2 \pi} \Delta G$. In such a fit we got (after integration) the negative sign of the gluonic contribution, i.e. opposite to the one expected from the paper [13]. Our conclusion is that the inclusion of gluon contribution does not lead to the substantial improvement of the fit. Encouraged by the present results we can make the next step and look for the differences in the structure of polarized sea for quarks and antiquarks (semi-inclusive hadron spin asymmetries differentiate these contributions). Similar suggestions were coming from model considerations [14]. As before we assume:

$$
\Delta M_{i}(x)=x^{-0.567}(1-x)^{10.1}\left(c+c_{i} \sqrt{x}\right),
$$

where i labels this time up, down, strange and charm quarks and up and 
down antiquarks. We have:

$$
\begin{aligned}
\Delta u_{M}(x) & =0.196 \Delta M_{u}(x)-0.5 \Delta \delta(x), \\
\Delta \bar{u}(x) & =0.196 \Delta M_{\bar{u}}(x)-0.5 \Delta \delta(x), \\
\Delta d_{M}(x) & =0.196 \Delta M_{d}(x)+0.5 \Delta \delta(x), \\
\Delta \bar{d}(x) & =0.196 \Delta M_{\bar{d}}(x)+0.5 \Delta \delta(x), \\
2 \Delta \bar{s}(x) & =0.196 \Delta M_{s}(x), \\
2 \Delta \bar{c}(x) & =0.020 \Delta M_{s}(x) .
\end{aligned}
$$

The data on semi-inclusive hadron spin asymmetries can not distinguish between $q$ and $\bar{q}$ for strange and charm quarks. The obtained fit is very similar to our basic fit. With two new parameters we have got $\chi^{2}$ only 0.5 smaller than before. The integrated quantities are very close to that gotten from eq.(8). The obtained sea contributions integrated in $0.003 \leq x \leq 1$ region are: $\Delta u_{M}=-0.04, \Delta \bar{u}=0.09, \Delta d_{M}=-0.02, \Delta \bar{d}=-0.07$ and $\Delta s=-0.12$.

It seems that with the data on semi-inclusive hadron asymmetries it is difficult to make definite statement about quark antiquark structure of polarized sea because all non-strange sea contributions are rather small and their signs depend on whether we use to fit all data points namely SMC and EMC or only SMC (of course together with inclusive data). More precise data are needed to make conclusive statement. When we use MRS fit A' the results practically do not change. The summed contributions for quark and antiquark are very close to the results obtained before and do not strongly depend whether we take for fitting SMC data only or SMC and EMC data.

We have extended our determination of polarized parton distributions by taking into account not only all available inclusive spin asymmetries but also semi-inclusive hadron spin asymmetries on protons and deuterons measured by experimental groups in CERN. By relaxing our previous assumption about the polarized sea we have found polarized sea contributions for up, down and strange quarks. The total sea contribution was strongly reduced. The results show stability and do not change when we make fit to inclusive data only or to all data (inclusive and semi-inclusive). There is also a very small change in calculated parameters when we use the other version of the MRS fit (called A') [7]. The attempt to determine quark-antiquark differences in the polarized sea structure do not seem to be conclusive because we have to 
do with the small numbers and the signs of different contributions depend on the subset of data used for fitting. The gluon contribution have sign opposite to the expected one and does not seem to influence the fit significantly. 


\section{References}

[1] B.Adeva et al. (Spin Muon Collaboration),Phys.Lett.B 369, 93 (1996);

[2] B.Adeva et al. (Spin Muon Collaboration), Phys.Lett. B 302, 533 (1993); D.Adams et al. (Spin Muon Collaboration), Phys.Lett. B 357, 248 (1995); D.Adams et al. (Spin Muon Collaboration), Phys.Lett. B 329, 399 (1994); D.L.Anthony et al. (E142 Collaboration), Phys.Rev.Lett. 71, 959 (1993); K.Abe et al. (E143 Collaboration), Phys.Rev.Lett. 74, 346 (1995); K.Abe et al. (E143 Collaboration), Phys.Rev.Lett. 75, 25 (1995); M.J.Alguard et al. (SLAC-Yale Collaboration), Phys.Rev.Lett. 37, 1261 (1976); G.Baum et al., Phys.Rev.Lett. 45, 2000 (1980); 51, 1135 (1983); J.Ashman et al. (European Muon Collaboration), Phys.Lett. B 206, 364 (1988); Nucl. Phys. B 328, 1 (1989);

[3] J.Ashman et al. (European Muon Collaboration), Nucl. Phys. B 328, 1 (1989);

[4] S.J.Brodsky, M.Burkardt, I.Schmidt, Nucl.Phys. B 441, 197 (1995); T.Gehrmann, W.J.Stirling, Z.Phys. C 65, 461 (1995); T.Germann, W.J.Stirling, Phys.Rev. D 53, 6100 (1996); F.Buccella et al., preprint Napoli DSF-T-95/26 (1995); C.Bourrely, J.Soffer, Nucl.Phys. B 445, 341 (1995); M.Glück et al. Phys.Rev. D 53,4775 (1996);

[5] J.Bartelski, S.Tatur, Acta Phys. Pol. B 26, 913 (1994); J.Bartelski, S.Tatur, CAMK preprint No. 288 to be published in Z.Phys.;

[6] J.Bartelski, S.Tatur, Acta Phys. Pol. B 27, 911 (1996);

[7] A.D.Martin, R.G.Roberts, W.J.Stirling, Phys.Rev. D 47, 867 (1993); Phys. Lett. B 306, 145 (1993); erratum B 309, 492 (1993); A.D.Martin, R.G.Roberts, W.J.Stirling, Phys.Rev. D 50, 6734 (1994);

[8] A.D.Martin, R.G.Roberts, W.J.Stirling, Phys.Lett. B 354, 155 (1995);

[9] W.L.Whitlow et al. Phys.Lett. B 250, 193 (1990);

[10] EMC, M.Arneodo et al. Nucl.Phys. B 321, 541 (1989) 
[11] S.A.Larin, Phys.Lett. B 334, 192 (1994);

[12] F.E.Close and R.G.Roberts, Phys.Lett. B 316, 165 (1993).

[13] G.Altarelli, G.G.Ross, Phys.Lett. B 212, 391 (1988);

[14] S.J.Brodsky, B.Q.Ma, SLAC-PUB-7126 (1996) 


\section{Figure captions}

Figure 1 The comparison of semi-inclusive spin asymmetries for production of positive (a) and negative (b) hadrons on protons with the curve gotten from our fit. Points are taken from SMC [1] and EMC [3] experiments.

Figure 2 The comparison of semi inclusive spin asymmetries for production of positive (a) and negative (b) hadrons (SMC data) with the curve gotten from our fit.

Figure 3 Our predictions for polarized quark distributions for : valence a) u, b) d quarks and sea c) u, d) d, e) s, f) $(u+d) / 2$ quarks. 
Figure 1a

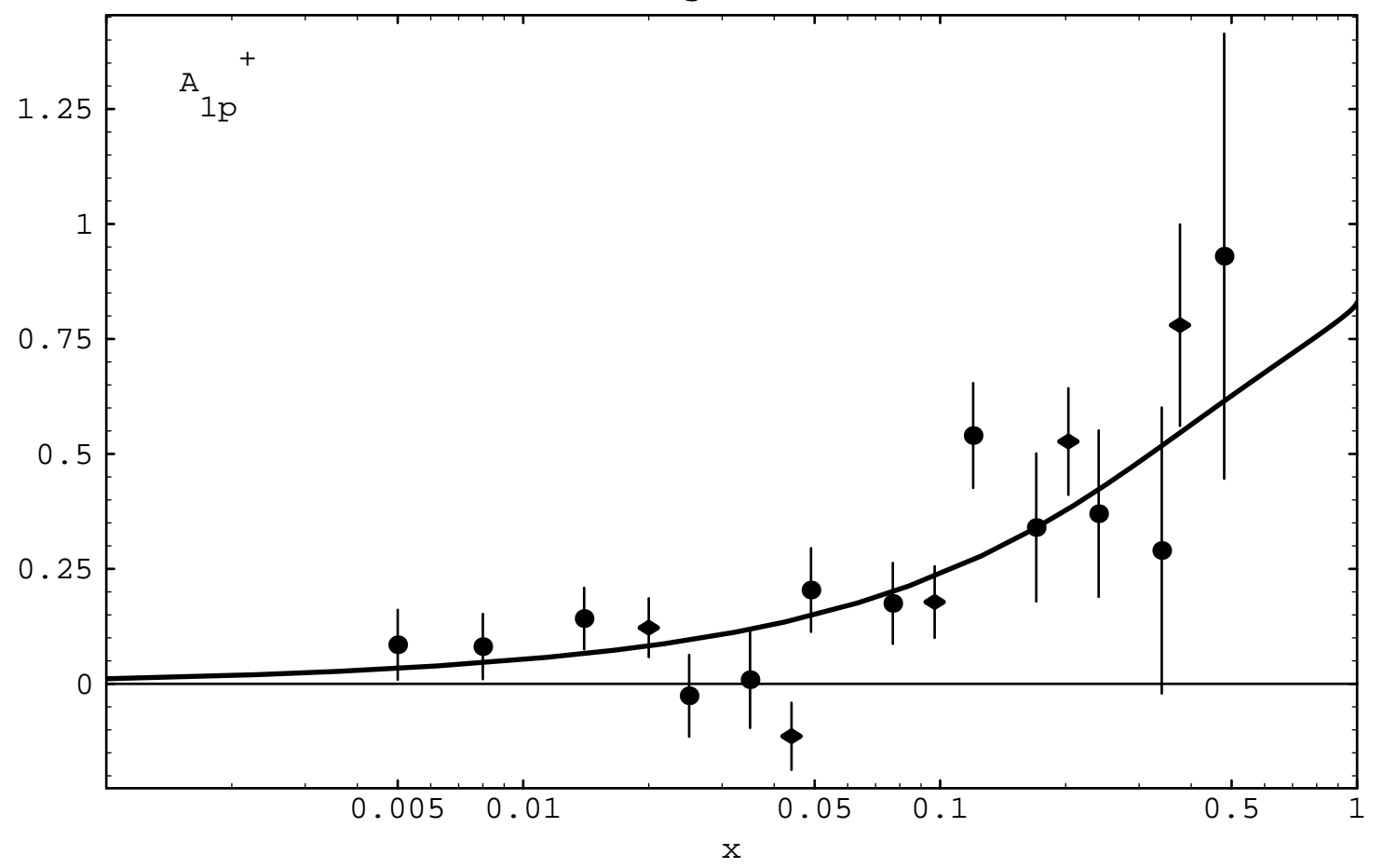

Figure 1b

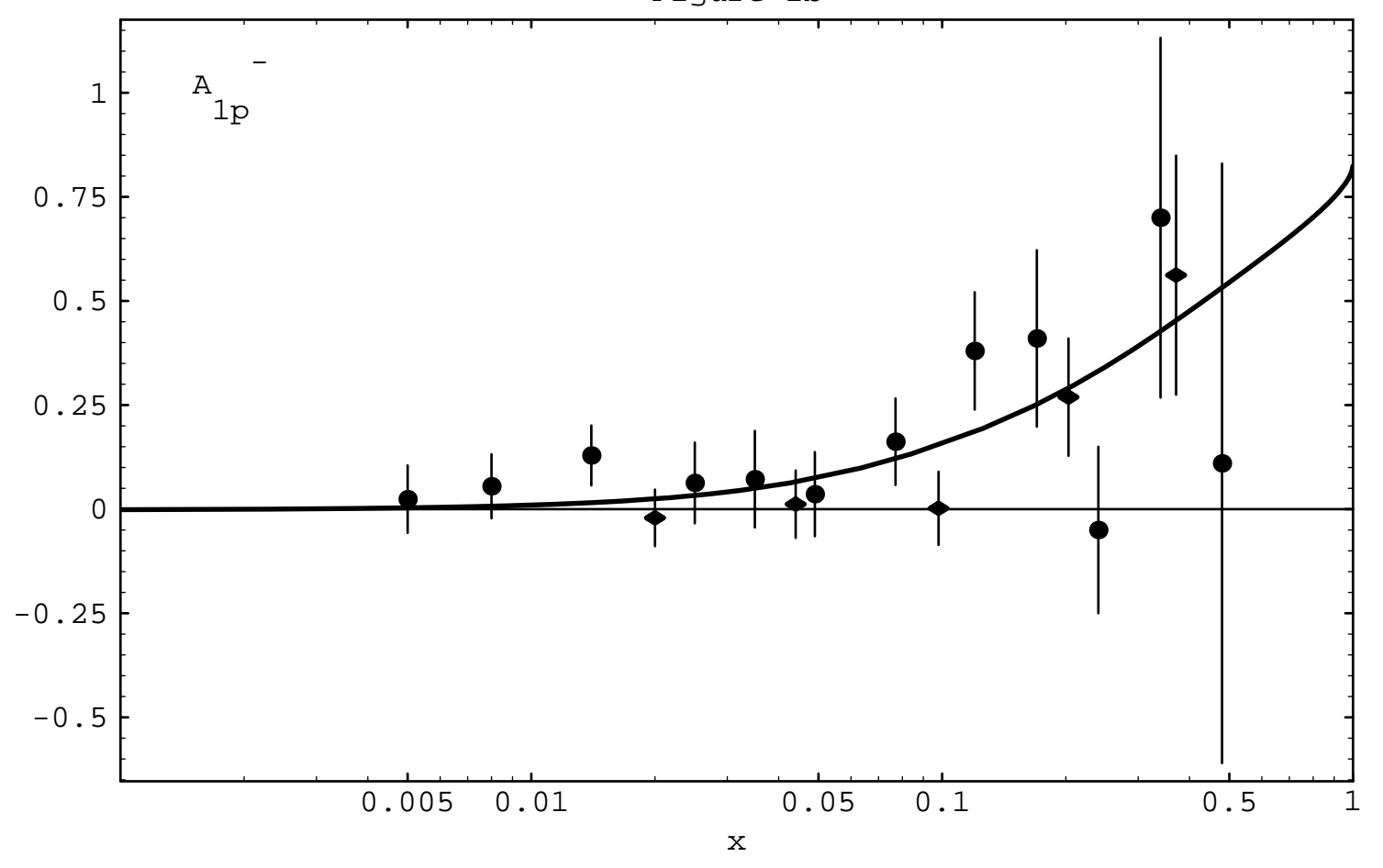


Figure $2 a$

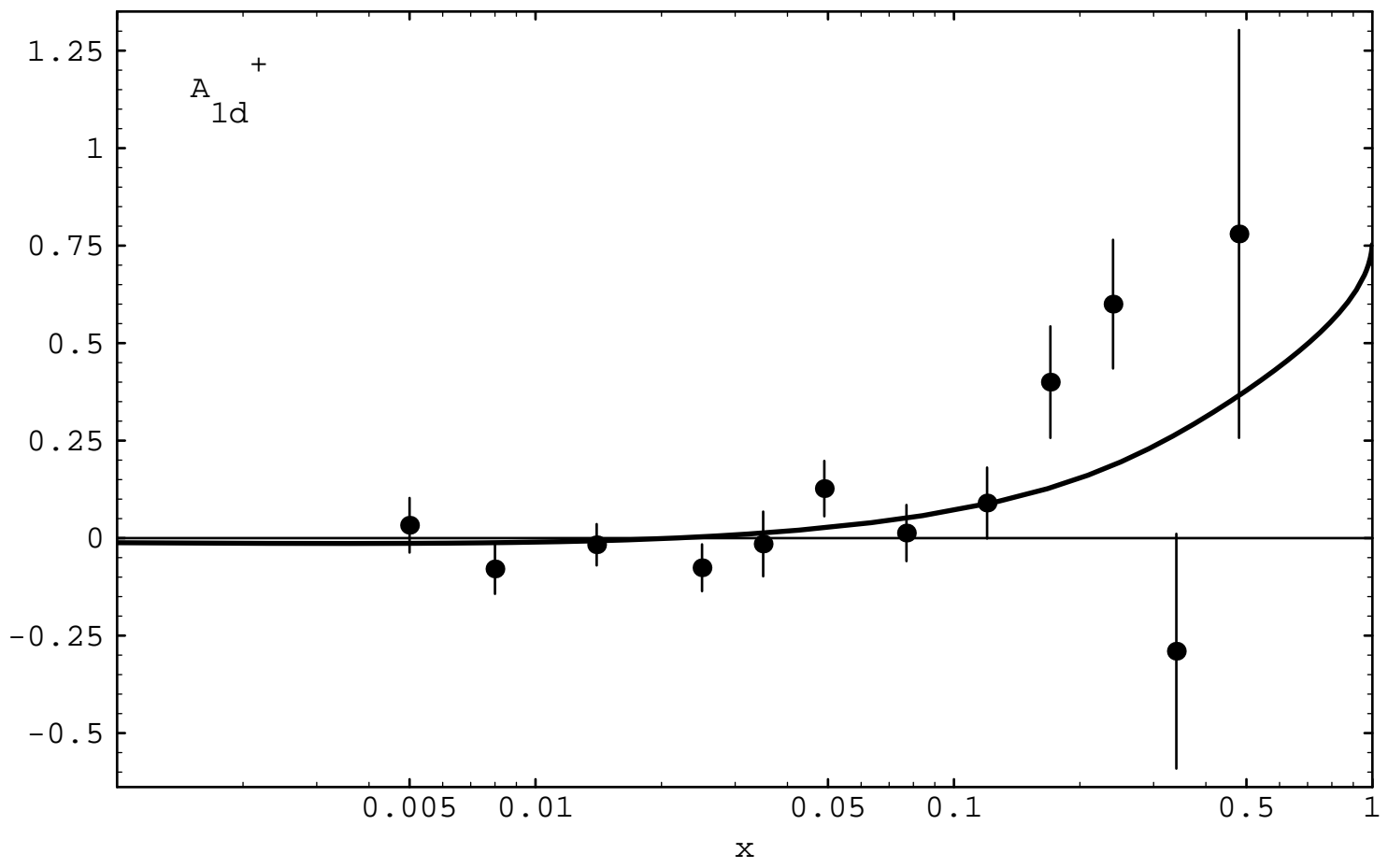

Figure 2b

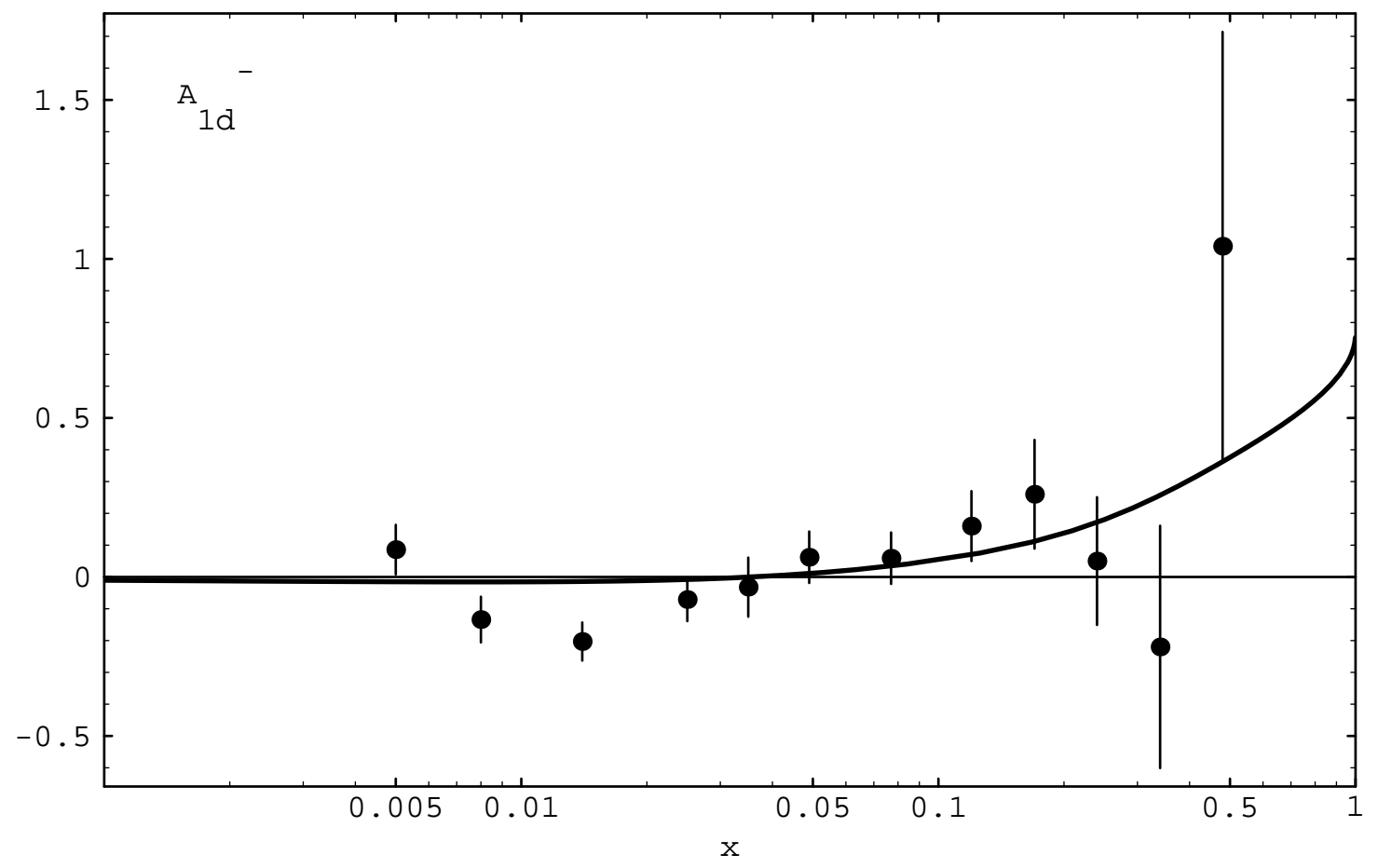


Figure $3 a$

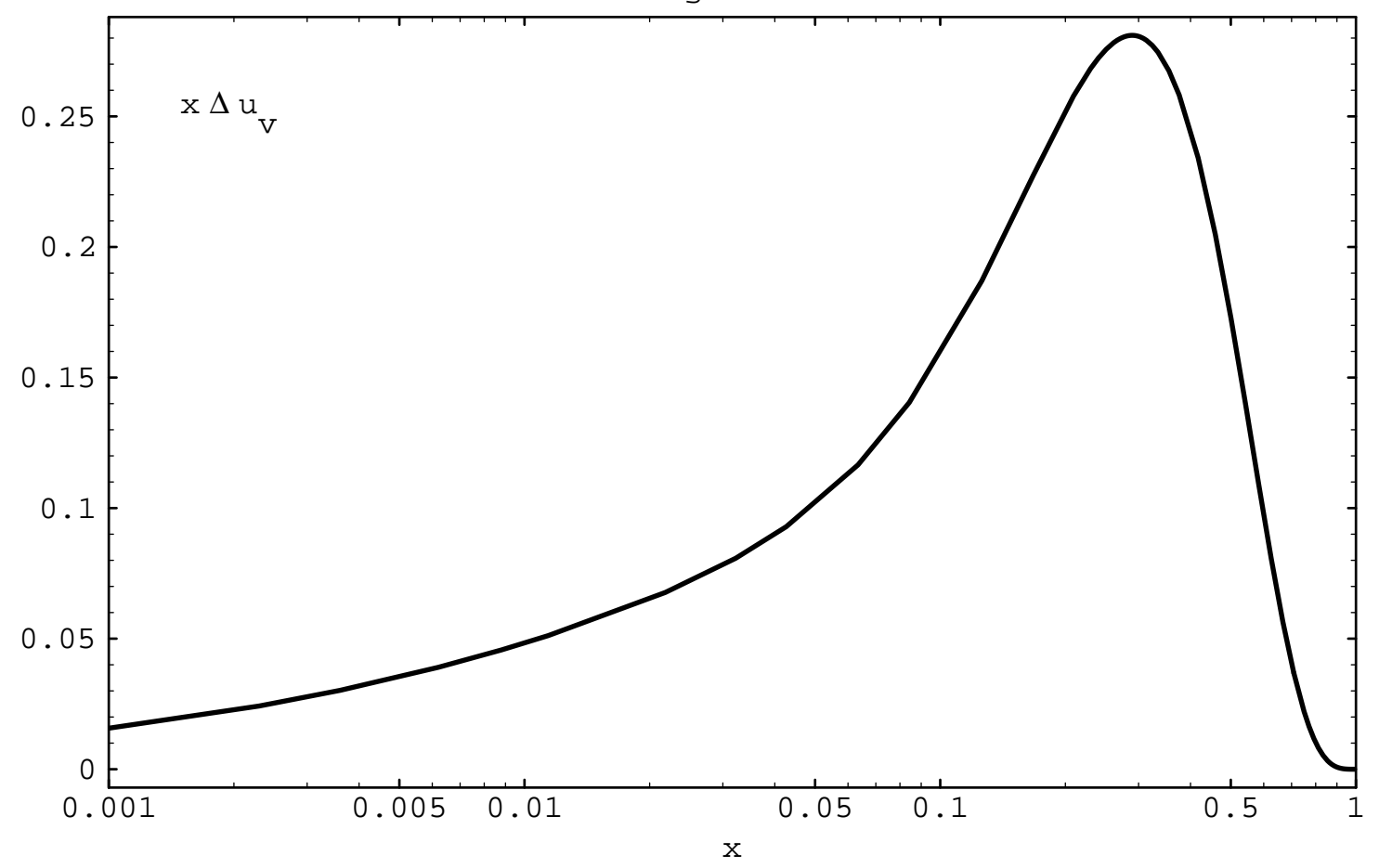

Figure 3b

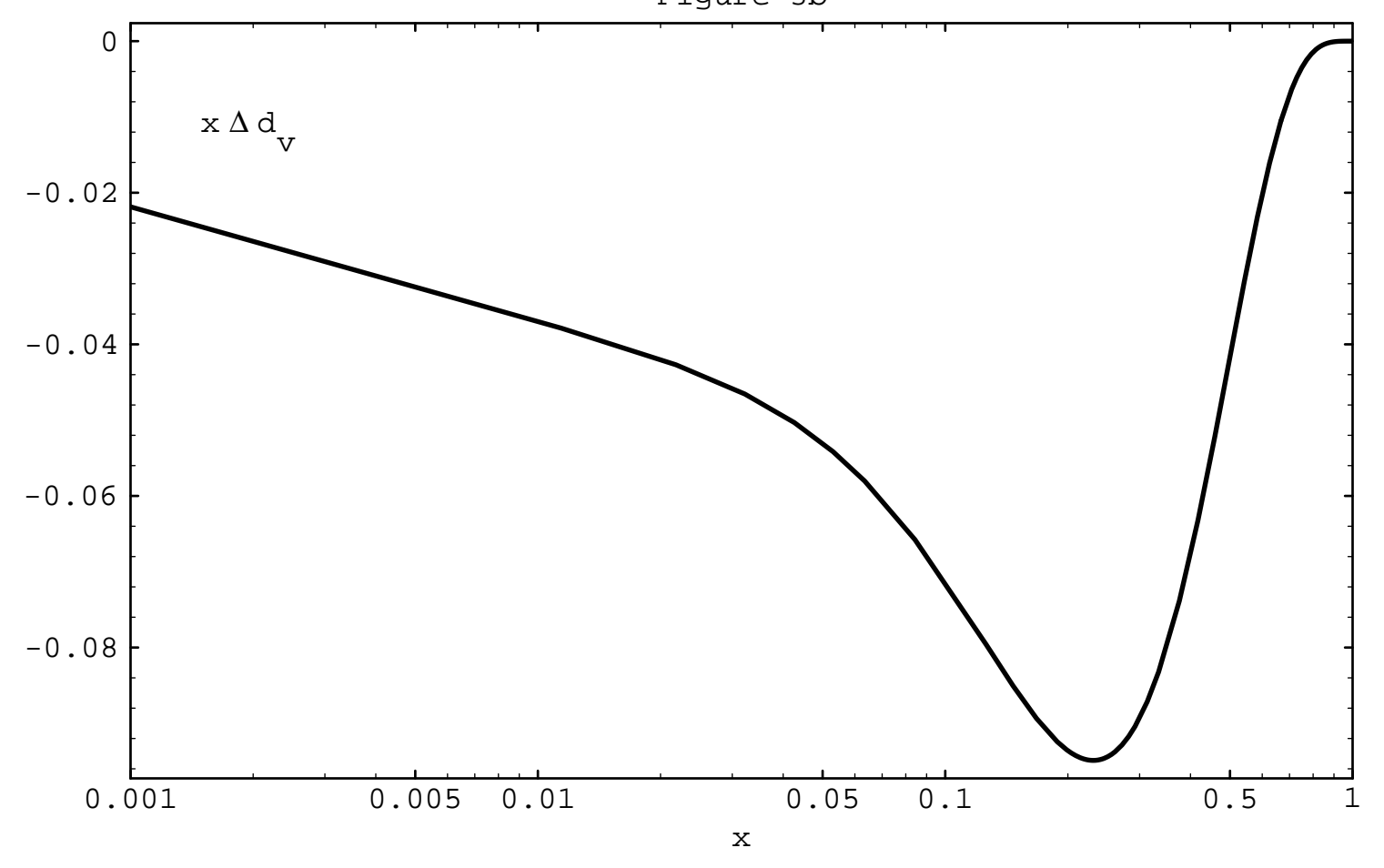


Figure 3c

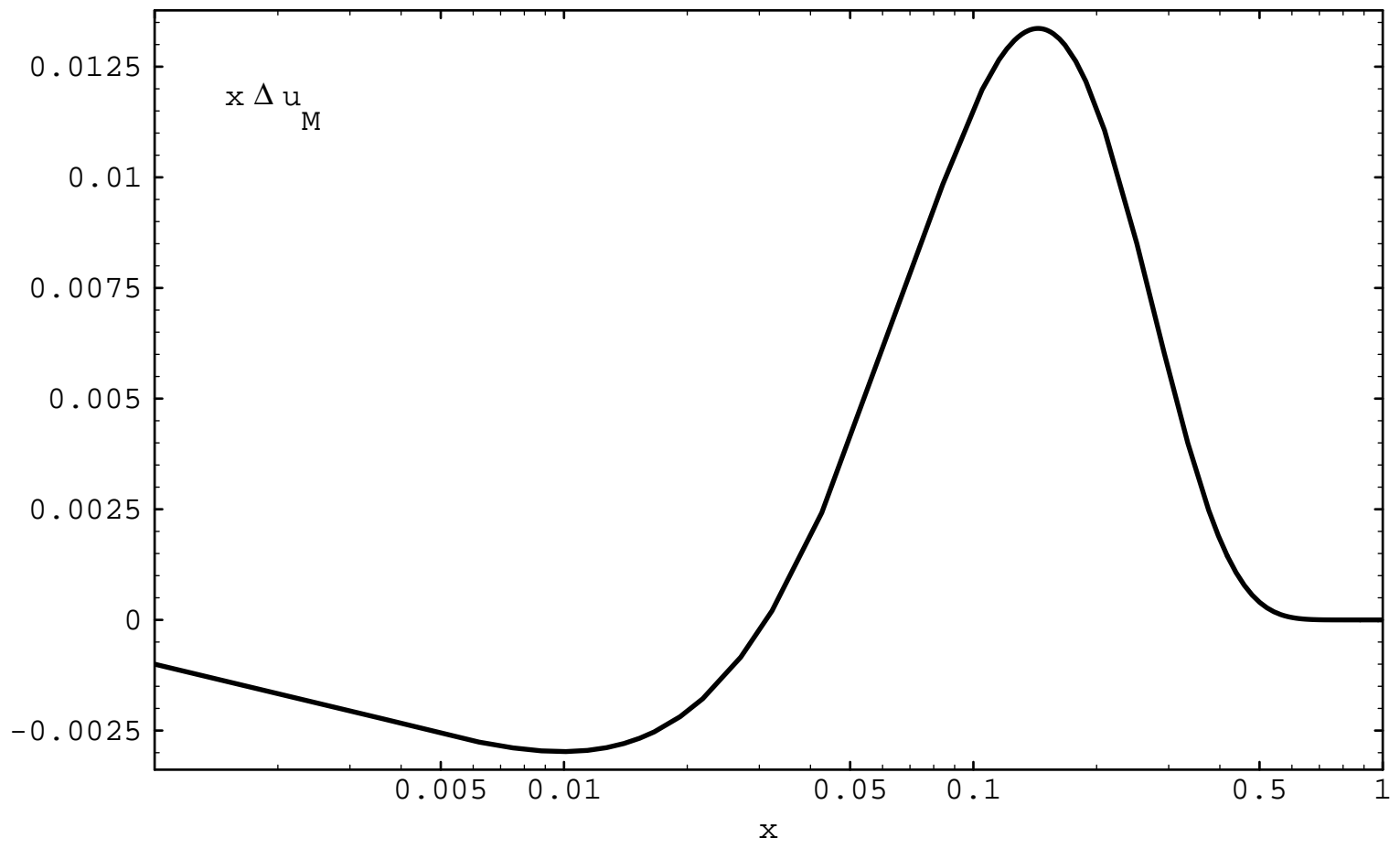

Figure 3d

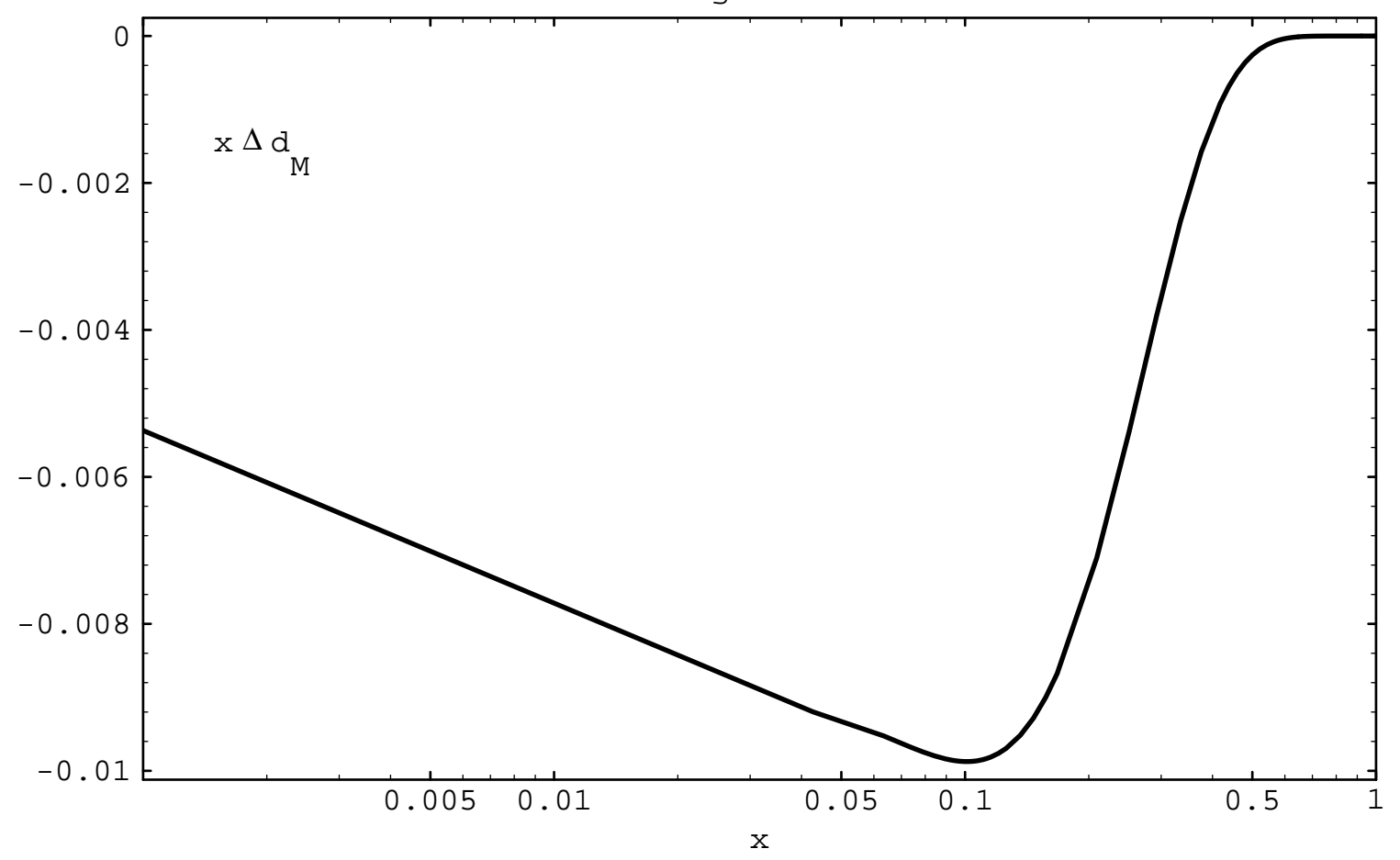


Figure $3 e$

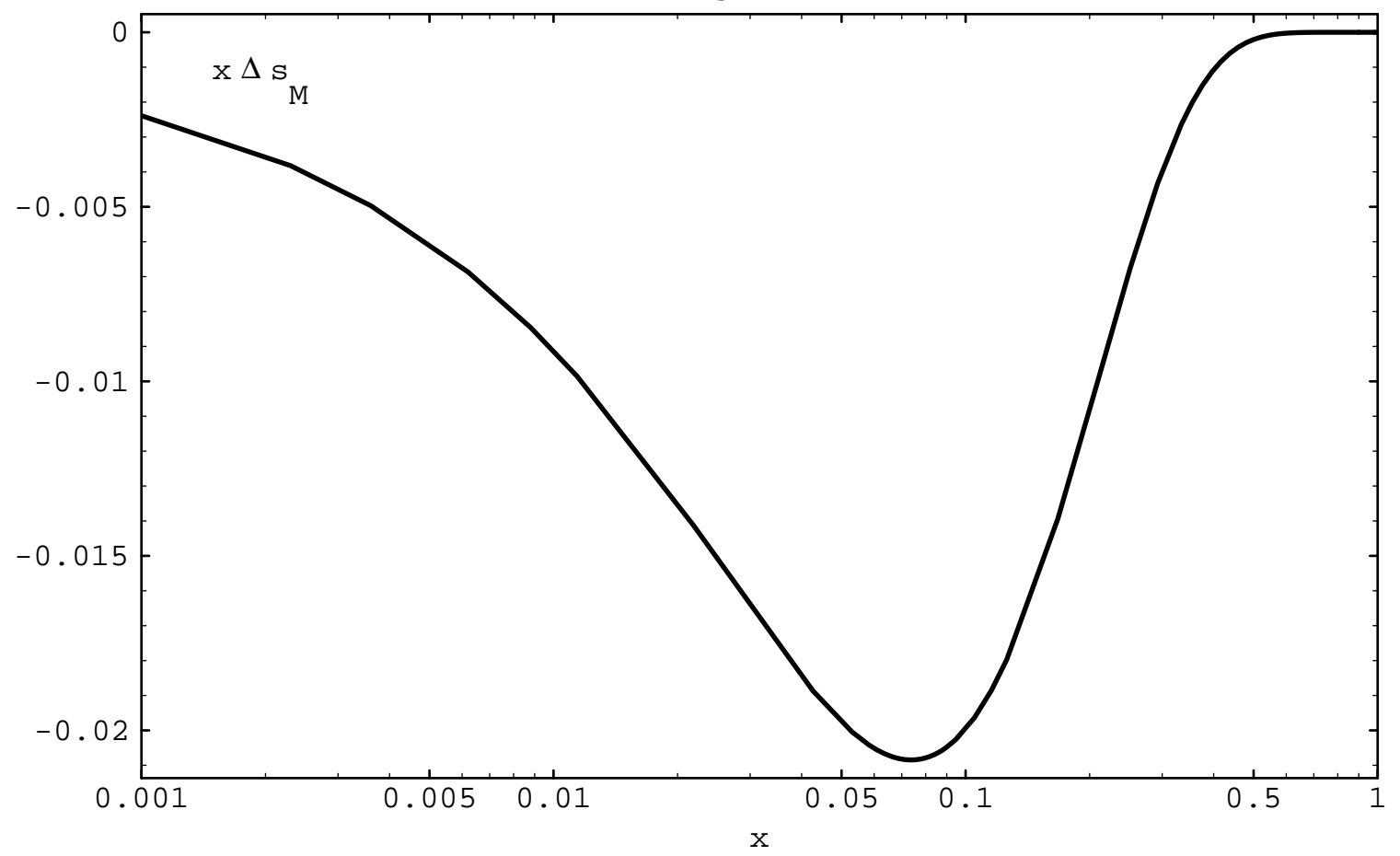

Figure $3 f$

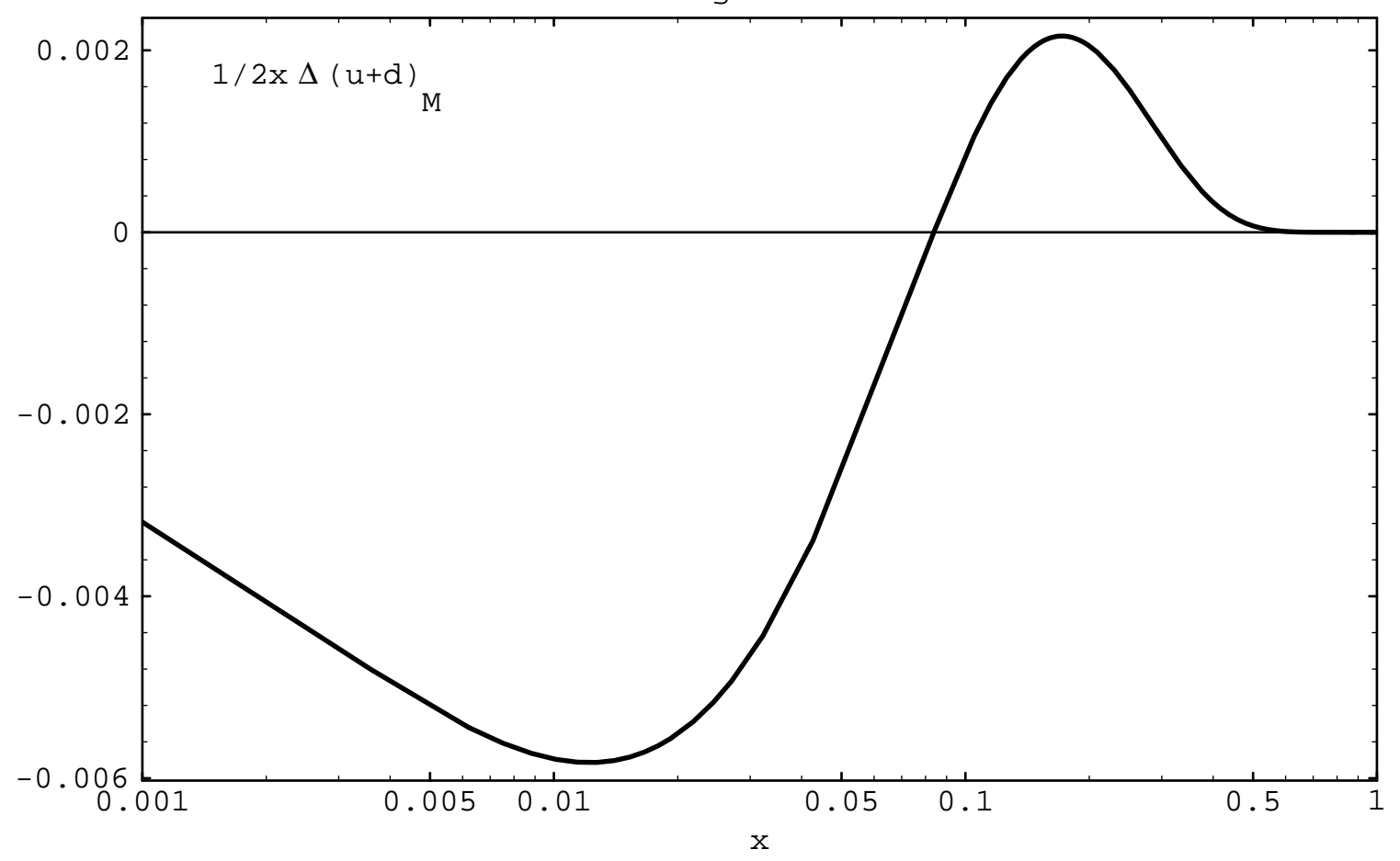

